\title{
Performance Increase of Lithium-Ion Batteries
}

\author{
A.V. Myatezh \\ Novosibirsk State Technical University \\ Novosibirsk, Russian Federation \\ user_m_82@mail.ru \\ B.V. Malozyomov
Novosibirsk State Technical University
Novosibirsk, Russian Federation
mbv5@mail.ru

\author{
M.A. Smirnov \\ Novosibirsk State Technical University \\ Novosibirsk, Russian Federation \\ smirnov@mail.ru \\ A.A. Shtang \\ Novosibirsk State Technical University \\ Novosibirsk, Russian Federation \\ shtangAA@mail.ru
}

\begin{abstract}
Lithium-ion battery (LIB) technology further enabled the information revolution by powering smartphones and tablets, allowing an unprecedented performance of these devices against reasonable cost. Currently, this battery technology is on the verge of carrying the revolution in road transport and energy storage of renewable energy. However, to fully succeed in the latter, a number of hurdles still need to be taken. Battery performance and lifetime constitute a bottleneck for electric vehicles as well as stationary electric energy storage systems to penetrate the market. Electrochemical battery models are one of the engineering tools which could be used to enhance their performance. These models can help to optimize the cell design and the battery management system. In this study, the authors evaluate the ability of the Porous Electrode Theory (PET) to predict the effect of changing positive electrode density in the overall performance of Li-ion battery cells. It can be concluded that Porous Electrode Theory (PET) is capable of predicting the difference in cell performance due to a changing positive electrode density.
\end{abstract}

Keywords-battery manufacturing, Li-ion, battery model.

\section{INTRODUCTION}

Today, more than ever, rechargeable batteries are everywhere in modern day-to-day life, serving as energy storage units in all sorts of devices. Li-ion Batteries (LIBs) represent the preferred battery technology for most applications owing to their superior performance [1, 2]. For a range of applications such as electric vehicles, electric boats and electric airplanes, they still lag behind when competing with established alternatives. This work is dedicated to tackling how far one can stretch the performance, as well as lower the cost of current LIB-technology? Overall battery performance is a result of many factors. The combination of development choices made during material selection, cell design, pack design and charging strategy [3] will impact the battery performance in the final use phase. Furthermore, the use of the phase itself will affect performance degradation. One can start to grasp the complexity of this optimization problem, mainly owing to the large number of input parameters spread over a number of blocks in the value chain (Figure 1). Not surprisingly, the battery industry relies mostly on a trial and error approach for their product development [4]. The lack of proper software tools to aid in this cumbersome task, as is done in many other areas of engineering, is due to the non-linear, complex, electrochemical nature of LIBs. The difficult experimental characterization of certain macroscopic active material properties and of the electrode matrix can be added to this.

A first step towards a workable simulation has been made already over two decades ago by Newman $[5,6]$. His model is a compromise between computational complexity and application relevance given that the model is able to take into account most important cell design properties at a low computational cost. Many authors have simplified or extended Newman's model. The approach of studying the macroscopic electrochemical behavior of porous electrodes is referred to as Porous Electrode Theory (PET) [7].

There is a great drive to keep reducing the price of LIBs during an enhanced performance. In this context, the impact of manufacturing procedures on the performance of battery modules has practically been disregarded in the literature. Past research on LIBs is fundamentally centered around materials and their properties on the cell or electrode level. In practice, many manufacturing parameters are chosen based on experience rather than analysis and calculation. Recent work has shown the potential gain in tuning and further developing LIB manufacturing [8]. For instance, in reference [9], the potential for a cost reduction of increasing the electrode thickness is demonstrated and, in reference [9], the influence of calendering on wettability is investigated. Initial and lifetime performance is strongly influenced by interventions in the manufacturing process, especially the morphology of the porous electrode [9].

In this publication, the influence of compression of the positive electrode (applied during the calendering step) on cell performance is experimentally investigated, keeping all other cell parameters constant. This step ensures an even electrode thickness and controls the electrode density. The electrode density is defined as the volumetric mass density of electrode material (the mixture of active material, a binder and conductive agent) in the electrode. In order to assess its 
impact, three different types of cells were made with different positive electrode densities. The active material in the positive electrode selected for this study was $\mathrm{Li}_{x} \mathrm{Ni}_{1 / 3} \mathrm{Mn}_{1 / 3} \mathrm{Co}_{1 / 3} \mathrm{O}_{2}$ (NMC). The choice of NCM is twofold: on the one hand, successful material optimization has made it superior to its predecessor $\mathrm{Li}_{\mathrm{x}} \mathrm{CoO}_{2}$ in multiple aspects, making it a relevant case to investigate. On the other hand, only limited research work has been conducted on multiphysics model of positive electrodes with NCM compared to lithium iron phosphate and lithium manganese oxide [10]. This work explains the different steps of LIB cell manufacturing, experimental details and results.

\section{THEORY}

The understanding of how the manufacturing of battery electrodes, cells, modules and packs influences battery performance is still in its infancy. Furthermore, system engineers are just starting to realize the impact of the battery management system's charge and discharge strategies [3]. Below, a short overview of the cell manufacturing process is given.

In the first step, Active Material (AM), binder (acting as a glue) and a conductive agent are mixed together in specific mass ratios to make the composite electrode (CE). The effect of the mixing sequence on the morphology of the $\mathrm{CE}$ and the electrochemical performances was discussed by Chang et al. [10]. They claim that the order of mixing should be optimized as a function of the constituents' properties, the nature of the solvent, and the viscosity of the dispersion. In order to compensate for the low AM conductivity, electron-conductive additives are generally employed to prepare CE for LIB. They can increase the available capacity, charge-discharge rate, and the cycle life performance of the cell. These electronconductive additives are mainly conductive carbon particles, such as acetylene black, Ketjen black, super-P, carbon graphite, carbon Nano-tubes etc.; however, these additives have a large surface area, which could lead to parasitic reactions with electrolytes [4]. The use of more fiber shaped carbons instead of more spherical Carbon Black (CB) enhances percolating properties of the electrode matrix [5].

Positive Electrodes (PEs) consist of Lithium metal oxide mixed with $\mathrm{CB}$ and polyvinylidene difluoride (PVDF). PVDF is dissolved in $N$-Methyl-2-pyrrolidone (NMP). Because of its toxic nature, relative low vapor pressures and high boiling point, there is a great drive to ban NMP from the manufacturing process. For the Negative Electrodes (NE), it has been successfully replaced by using a carboxymethyl cellulose-based binder in combination with styrene butadiene rubber. In this case, water can be used as a solvent. Today, in the majority of LIBs, carbon is used as AM for the NE [6]. CB can also be added to the NE to improve conductive pathways and the porous structure. The conduction network is formed by the CB particles, which fill the void between AM particles and are interconnected by particle-to-particle point contacts from the current collector to the most outer AM layer. The electronic transfer at the $\mathrm{CB} / \mathrm{CB}$ contact points is due to the tunneling effect phenomenon. Dominko [7] demonstrated that a good carbon coating with a homogeneous distribution can decrease the amount of $\mathrm{CB}$ to $2 \mathrm{wt}$. $\%$ and also increase the kinetics of the resulting electrode when compared to a conventional electrode. It presents an opportunity for cell manufacturers to tune their cell performance. Especially for badly conducting AM, the influence of binders and conductive agents can be very influential [5].

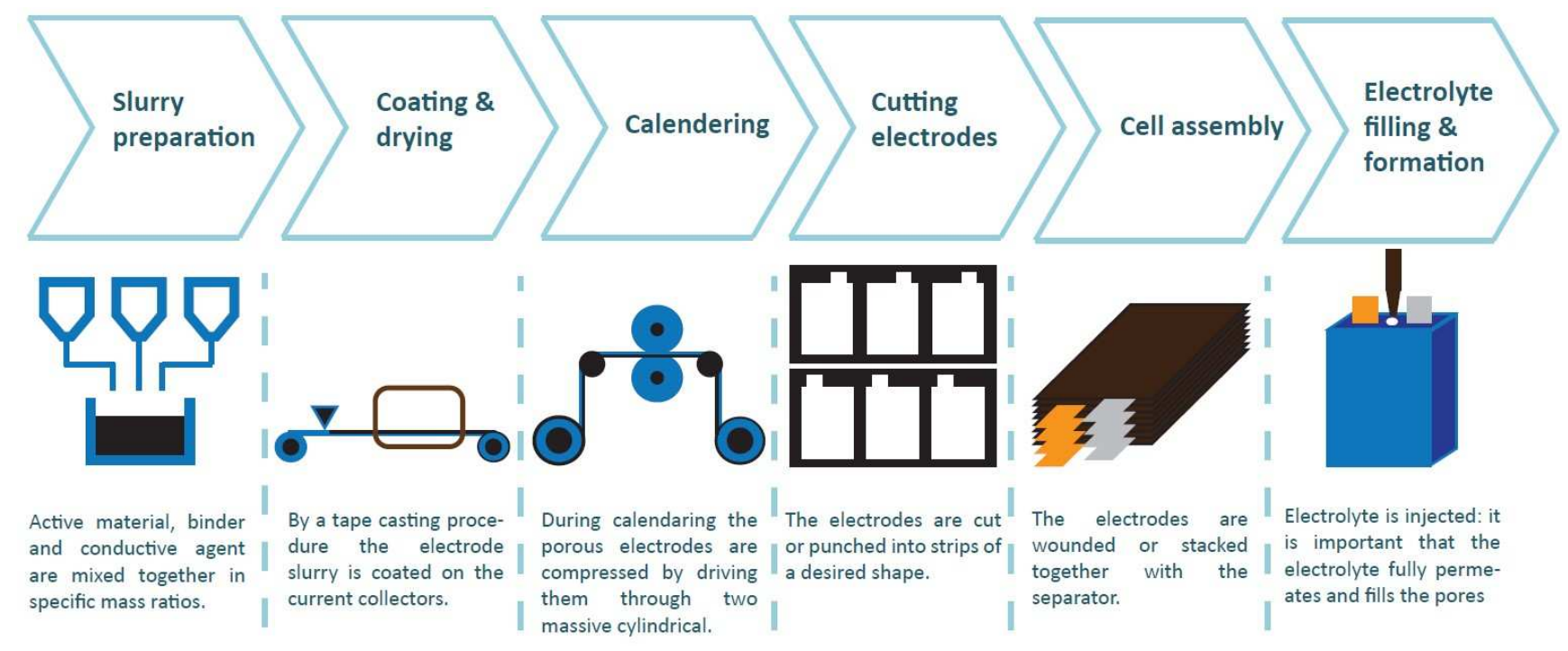

Fig. 1. Manufacturing steps of Li-ion batteries

In addition, the solvent concentration is an important factor in the process and standard formulation of CEs. For a concentration below the optimal one, the electrode dispersion exhibits a yield stress that inhibits flow and prevents homogeneous distribution of the constituents. Above the optimal concentration, settling of the low viscosity dispersion can create a concentration gradient. Thus, the solvent concentration (or solid loading) has a strong impact on the 
morphology and consequently on the electrochemical performance of the CE. Below and above the optimal solvent concentration, the electrochemical behavior is degraded due to poor electronic wiring of the AM [8].

The electrode slurry (or paste) is coated by a tape casting procedure on the current collectors: for the NE, this is copper, and for the PE, this is aluminum. During this process, it is required to control the solvent concentration. Once powders are dispersed, the slurry will be spread onto the current collector using the doctor blade with a chosen thickness. The tape cast should be dried to eliminate the residual solvents. In this step, it is important to obtain a homogenous coating thickness. The amount of active material per $\mathrm{cm}^{2}$ of the current collector determines the Maximum Available Capacity (MAC) of the electrodes. Both electrodes should be balanced out well so that the desired voltage window of both electrodes is actually used. Furthermore, one has to take into account irreversible capacity loss because of SEI formation, mostly on the graphite-based NE. Given that the cell is assembled from unlithiated carbon and fully lithiated Lithium metal oxide, a discharged state, the PE is not optimally used. After coating, the electrodes are passed through an oven to dry out the solvent: NMP for the PE and water for the NE.

During calendaring, the electrodes are compressed by driving them through two massive cylindrical rolls. By applying large pressure $\left(300-2000 \mathrm{~kg}^{*} \mathrm{~cm}^{-2}\right)$, the electrode thickness is reduced to a controlled value. The porosity is reduced by around $40 \%$ [9]. As a result, the adhesion of electrode materials is improved and the density is increased. The effects on battery performance are discussed in the next paragraph. After this operation, the electrodes can be dried for a second time to remove all water contaminations. The rest of the manufacturing process will take place in a dry room.

Then the electrodes are cut or punched into strips of a desired shape. A clean cut is necessary to avoid burrs on the edges which can cause short-circuit in the cells. Let us note that NEs are made slightly larger than PEs to avoid lithium deposition and dendrite formation on the edge of the NE. A large oversizing of the NE leads to a loss of energy density of the complete cell. Contacting tabs are fixed to the electrode.

The electrodes are wounded or stacked together with the separator. The electrode stack or jelly roll is integrated into the housing. To evacuate gases which arise during the formation, the housing is partially sealed.

Finally, electrolytes are injected. To achieve a maximal wettability, it is important that the electrolyte completely permeates and fills the pores in the separator and electrode [10]. Un-wetted AM area will decrease the specific surface area, which is reacting during battery operation and, as result, increases the cell impedance. Furthermore, wettability is important to achieve a uniform SEI layer during the formation cycles. The calendaring step will inevitably influence the wettability because it alters the porosity and particle distribution [8]. The wetting formation-step takes up lot of time (1.5 to three weeks) and capital [8].
If the chemical nature of all components is fixed, one still has the ability to vary the following process parameters over a wide range:

\section{Particle size and distribution.}

2. Relative amount of AM, a binder and conductive agent in the electrode.

3. Amount of electrode material AM per $\mathrm{cm}^{2}$ of the current collector.

\section{Thickness and density of the electrode.}

\section{Salt concentration of the electrolyte.}

6. Wettability process, determining active area which all have non-accurately known impact on life time and battery performance. In the next paragraph, the influence point three on the PE is investigated.

In order to assess the influence of PE density three times, 10 cells with a $\mathrm{Li}_{x} \mathrm{Ni}_{1 / 3} \mathrm{Mn}_{1 / 3} \mathrm{Co}_{1 / 3} \mathrm{O}_{2}$ based $\mathrm{PE}$ and graphite based NE were made. The Open-Circuit-Potential (OCP) of the electrodes versus lithium metal used in the simulations can be found in reference [10]. The first group of 10 cells had a PE density of $3.2 \mathrm{~g} / \mathrm{cm}^{3}$, the second group $-3.0 \mathrm{~g} / \mathrm{cm}^{3}$ and the third group $-2.8 \mathrm{~g} / \mathrm{cm}^{3}$. For the three types of cells, the electrode formulation of both electrodes was the same. In the rest of this paper, the three types of cells will be referred to as High Density (HD), Middle Density (MD) and Low Density (LD). Let us notice that the high density electrodes have the lowest volume fraction of electrolyte (also known as porosity). The 1C current rate of the cells is fixed at $600 \mathrm{~mA}$ or 18.5 $\mathrm{A} / \mathrm{m}^{2}$. For the purpose of generality, from here on all capacities and current rates will be expressed per $\mathrm{m}^{2}$ of PE.

An LIB can be considered as a voltage source with a nonlinear time varying impedance. If one was able to calculate this impedance based on the material and design specifications of the cell, this will enable the battery designers to optimize the cell design for a given application and corresponding realtime operation. The impedance in a porous LIB electrode is a mixture of multiple physical processes [2]:

- Electric conduction at the current collector/electrode interface results in a resistive contact impedance. In this regard, it is important to stress the effect of deformation caused by the pressure, applied during the calendering procedure. It can be seen that the pressure of the active material on the aluminum current collector deforms this interface creating a better electric contact and thus lower resistance.

- Electric conduction in the matrix of the porous electrode depends on the intrinsic conductivity of the AM, this electronic current is mostly carried by the carbon-binder domain (CBD) [5].

- Mass transfer (mostly diffusion) of lithium ions in the intercalation host [2].

- The total current passing through the electrode/electrolyte interface is the sum of a capacitive current and faradaic current.

- The capacitive current is due to the electric double layer, formed at the electrode/electrolyte interface. In batteries in 
general and in LIBs specifically, this current is considered small relative to the total current [3].

- The faradaic current is due to the charge transfer reaction accompanied with Li-intercalation.

- Ionic conduction in the electrolyte.

So what can be expected of an increased (PE) electrode density? The higher pressure which is used during calendering results in a better contact between the electrode matrix and the aluminum foil, decreasing the contact resistance. HD electrodes have a higher volume fraction of CBDs in the pore space resulting in better binding and conduction between the particles. These two effects should decrease the overall cell impedance, however the magnitude of the effect depends on the nature of the AM and CBDs [5].

Higher electrode density will result in less available active surface area of the AM. The volume fraction of electrolyte (or porosity) decreases as the electrode density increases. In addition, low porosity has a deteriorating effect on electrolyte transport. In this regard, tortuosity can also be influenced badly. The extent of these effects can be investigated with advanced electrode imaging techniques such as those presented by Hutzenlaub [5]: using a combination of X-ray tomography to depict the AM domain, and Focused Ion Beam Scanning Electron Microscope (FTB/SEM) images to obtain a phase distribution of AM, CBD and pore space, they are able to establish a full digital 3D image of the electrode.

There is a mix of beneficial (electric conductivity) and deteriorating (specific surface area and electrolyte transport) effects to the cell impedance. Experimentally, one will assess the overall effect on the cell impedance in the rest of the paragraph. The extent of the overall effect can be low because individual effects compensate each other and/or because the $\mathrm{NE}$ is dominating the total cell impedance.

\section{EXPERIMENT}

After formation and preconditioning tests, the cells were subjected to a series of identical characterization tests. First, the Maximum Available Capacity (MAC) and Maximum Available Energy (MAE) between the recommended maximum and minimum voltage were measured, respectively $V_{\max }=4.2 \mathrm{~V}$ and $V_{\min }=2.7 \mathrm{~V}$. This test regime is as follows: after a Standard Charge Regime (SCR) the cells are discharged with a $\mathrm{C} / 25$ constant current until $V_{\text {min }}$. After a resting period of one hour, the cells are charged with the opposite charge profile until $V_{\max }$. From this test, respectively, two values for the MAC and MAE are obtained: one - for the charge, and one - for the discharge regime. The SCR is defined as a constant current charge of $\mathrm{C} / 2$ until $V_{\max }$, then a constant voltage charge reaches $\mathrm{C} / 100$ followed by a relaxation period of one hour. Secondly, the available discharge capacities between $V_{\max }$ and $V_{\min }$ at multiple current rates were determined. These are obtained by discharging the cells after an SCR with a constant current of, respectively, $\mathrm{C} / 2,1 \mathrm{C}, 2 \mathrm{C}, 3 \mathrm{C}, 4 \mathrm{C}$ and 5C. Thirdly, a pulse characterization test was applied to determine the Direct Current Resistance (DCR) and to calculate the power capability of the cells. The pulse characterization test starts with an SCR, after which the cells are discharged with a $\mathrm{C} / 2$ rate in blocks of $10 \%$ of the rated capacity. Then, alternating discharge and charge pulses of $3 \mathrm{C}$ were applied. The relaxation period in between pulses was $15 \mathrm{~min}$.

The DCR $\left(R_{D C R}\right)$ was determined by fitting the voltage response to a first order model minimizing the sum of the squares of the errors. The details of this procedure can be found in reference [6]. During all experiments, the cells were put in a climate chamber keeping the surrounding temperature of the cells at $25^{\circ} \mathrm{C}$.

As can be seen from Table 1, the MAC of the fresh cells is as expected and within acceptable error margins, the same for the three cell types, being $(20.8 \pm 0.1) \mathrm{Ah} / \mathrm{m}^{2}$ for the discharge regime, and $\left(20.9 \pm 0.1 \mathrm{Ah} / \mathrm{m}^{2}\right)$ - for the charge regime. The MAE is $(77.1 \pm 0.3) \mathrm{Wh} / \mathrm{m}^{2}$ for the discharge regime, and $(77.5 \pm 0.3) \mathrm{Wh} / \mathrm{m}^{2}$ - for the charge regime. In Figure 2, the discharge capacities are given relative to the MAC. As can be seen, at low current rates $(0.5 \mathrm{C}$ and $1 \mathrm{C})$, the HD cells have a slightly higher capacity of around $0.4 \%$, compared to the LD cell type. At $2 \mathrm{C}$ and $3 \mathrm{C}$, the difference is lower and the trend is broken. At these current rates, the discharge capacities do not show a significant difference between LD, MD and HD cell types. High current rates of $4 \mathrm{C}$ and $5 \mathrm{C}$ show significant differences, respectively $2.7 \%$ and $8.9 \%$. The difference in available energy at the different current rates is similar. Furthermore, one can note that for a $3 \mathrm{C}, 4 \mathrm{C}$ and $5 \mathrm{C}$ current rate, $\mathrm{MD}$ and $\mathrm{HD}$ cell types show and increased standard deviation, which indicates a bigger spread of measured discharge capacities in the respective groups.

TABLE I. RESULTS OF THE MEASUREMENT OF THE MAXIMUM AVAILABLE CAPACITY (MAC) AND MAXIMUM AVAILABLE ENERGY (MAE) FOR THREE CELL TYPES.

\begin{tabular}{|c|c|c|c|c|c|c|c|}
\hline \multirow{2}{*}{ Measured Quantity } & \multirow{2}{*}{ LD } & \multicolumn{3}{|c|}{ MD } & \multicolumn{2}{c|}{ HD } \\
\cline { 3 - 8 } & & Dis & Cha & Dis & Dis & Dis & Cha \\
\hline \multirow{2}{*}{ Maximum Available Capacity $\left(\mathrm{Ah} / \mathrm{m}^{2}\right)$} & Mean & 20.81 & 20.82 & 20.84 & 20.87 & 20.84 & 20.87 \\
& St. dev. & 0.09 & 0.07 & 0.07 & 0.05 & 0.12 & 0.10 \\
\hline \multirow{2}{*}{ Maximum Available Energy $\left(\mathrm{Wh} / \mathrm{m}^{2}\right)$} & Mean & 77.23 & 77.54 & 76.92 & 77.54 & 77.28 & 77.55 \\
& St. dev. & 0.32 & 0.26 & 0.25 & 0.20 & 0.42 & 0.37 \\
\hline
\end{tabular}

The measurements of the DCR, based on a current pulse profile, is given in Figure 3 for charge and discharge. The average DCR of all cells was taken, and the width of the error bars equals two times the standard deviation. For reasons of clarity, only the results of the DCR for the HD and LD cell types are depicted. For all cell types, there is a decreasing trend as the SoC increases. The difference in DCR between the HD and LD cell types is quite constant over the considered SoC window. LD cell types have a larger DCR: on average, it is $0.116 \mathrm{~m} \Omega \cdot \mathrm{m}^{2}$ larger for 
discharge pulses and $0.097 \mathrm{~m} \Omega \cdot \mathrm{m}^{2}$ - for the charge pulses. Expressed relative to the lowest value, the difference is on average $5.1 \%$ for discharge and $4.3 \%$ - for charge. Except for low SoC values, charge and discharge pulses result in the same DCR. At low SoC values, discharge pulses give a higher DCR. The relatively large spread among the DCR measurements is caused by: cell to cell variations, slight temperature variations depending on the position in the climate room, systematic differences in the experimental setup and measurement, and variations in post-processing of the data.
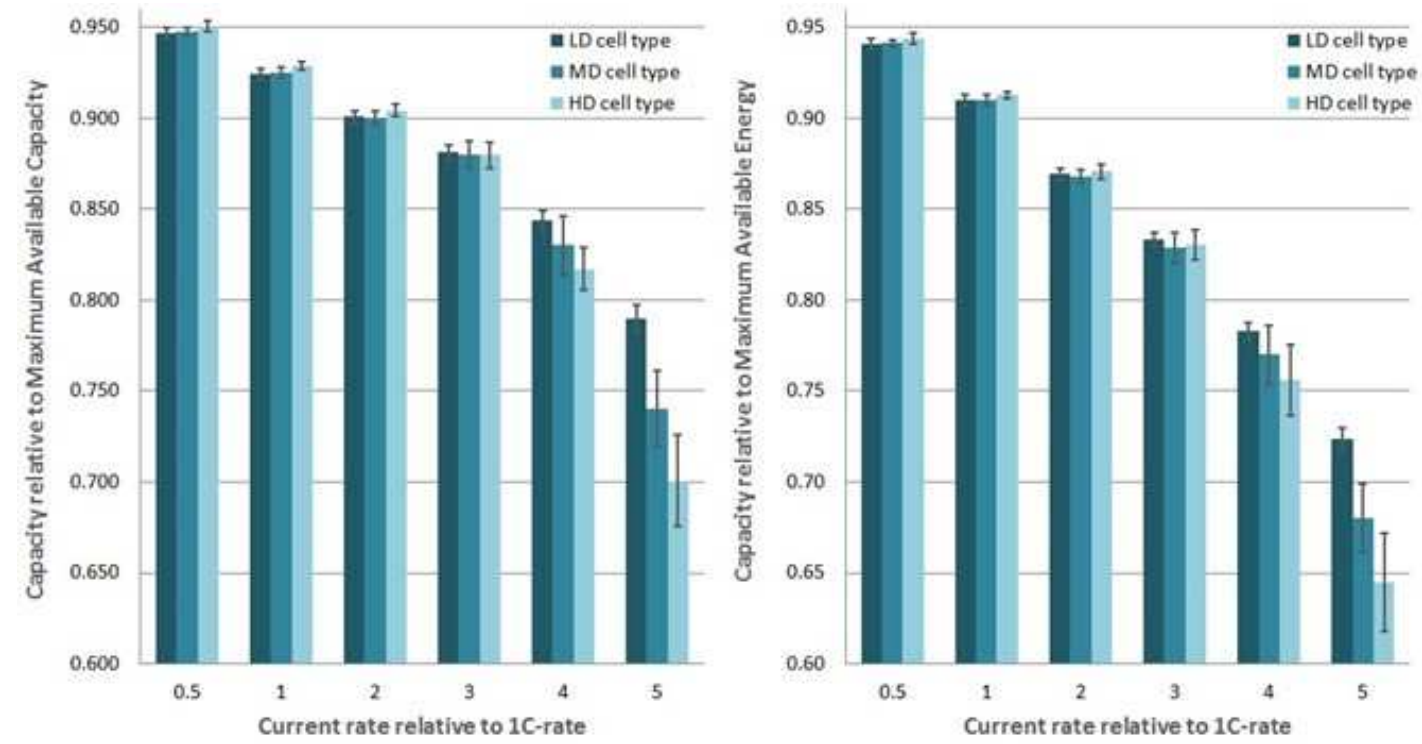

Fig. 2. Influence of the positive electrode density on the discharge capacity at multiple current rates. HD stands for High Density, MD - for Middle Density and LD - for Low Density. The capacity is given relative to the MAC. The width of the error bars is twice the standard deviation

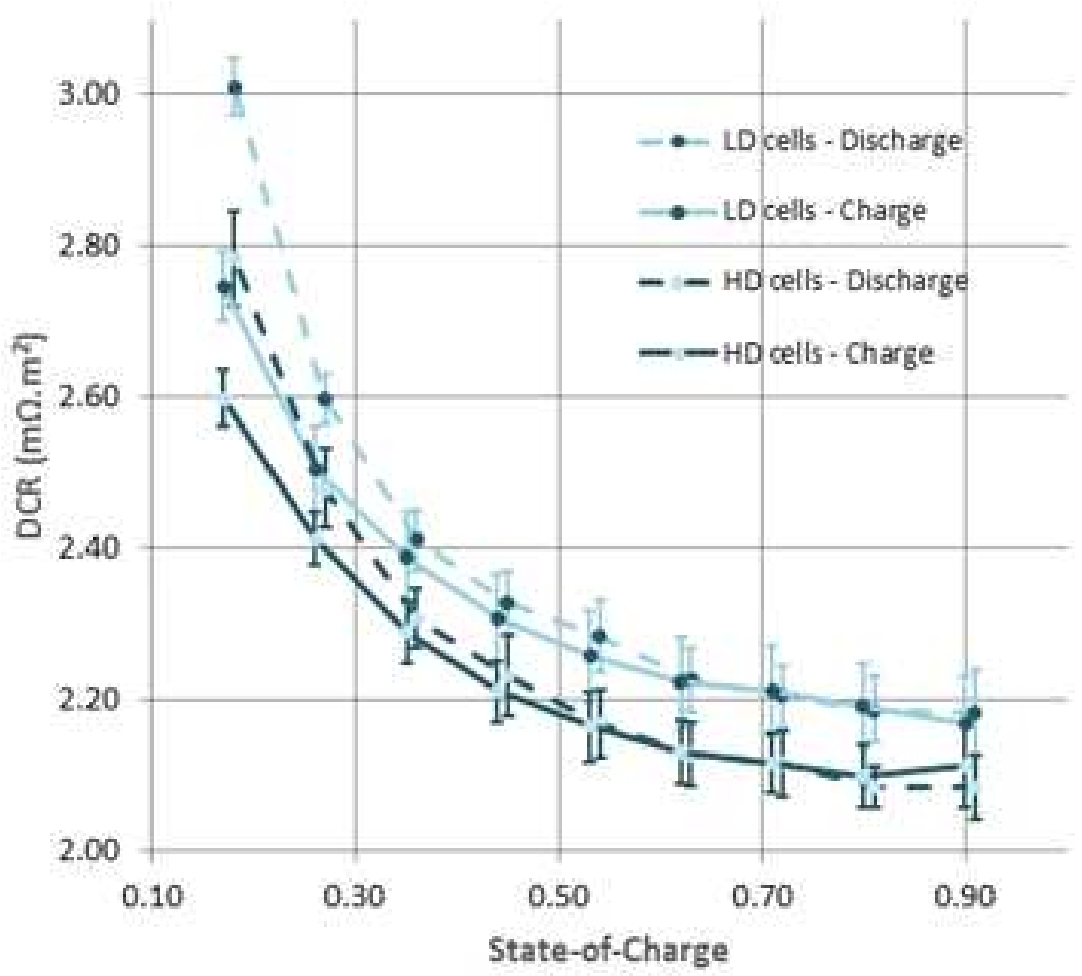

Fig. 3. Influence of the positive electrode density on the Direct Current Resistance at multiple SoC levels. HD stands for High Density and LD - for Low Density. The ohmic cell resistance (or DCR) is given per $1 \mathrm{~m}^{2}$ of a positive electrode. The width of the error bars equals two times the standard deviation 


\section{CONCLUSION}

To continue to improve the performance of state-of-theart LIBs, accurate battery models are required.

These models should provide reliable prediction in the function of material parameters and battery manufacturing parameters. If done so, based on an accurate scientific frame work, the results can be extrapolated over a wide range of material and manufacturing parameters, enabling battery and system designers to find the best solutions for their applications. From this work, one concludes that PET shows good promise to simulate LIBs at reasonable computational cost.

This work investigated the applicability of PET for breaking down the impacts of manufacturing processes on the dynamic performance of LIBs. The effect of one manufacturing parameter, the density of the PE, was experimentally investigated for an NMC/graphite based cell. Cells with high PE density show a slightly higher discharge capacity at low current rates. At high current rates, cells with a low PE density clearly show a better performance. The authors would like to stress that this effect is likely to be more pronounced at lower rates if the electrodes were made with more AM per $\mathrm{cm}^{2}$ of electrode (higher loading, thicker electrodes or, in other words, more energy dense cells). When observing the DCR, high density PEs are better, showing a $4 \%$ to $5 \%$ lower DCR.

The PET model was capable of predicting the relative difference of the effect PE density for the measurement of the DCR, as well for measurement of the discharge capacity. To achieve an accurate validation over the whole set of experimental data remains cumbersome.

\section{References}

[1] W. Jarzyna, D. Zieliński, P. Hołyszko, "Battery-supported trolleybus traction network - a component of the municipal smart grid", Environmental Engineering V Proceedings of the 5th National Congress of Environmental Engineering, pp. 287-292, 2017.

[2] Y. Liao, Z. Liu, G. Zhng, C. Xiang, "Vehicle-Grid System Modeling and Stability Analysis with Forbidden Region-Based Criterion", IEEE Transactions on Power Electronics, vol. 32, iss. 5, pp. 3499-3512, 2017

[3] I. Maghsoud, A. Ghassemi, S. Farshad, S. S. Fazel, "Current balancing, reactive power and harmonic compensation using a traction power conditioner on electrified railway system", 21st Iranian Conference on Electrical Engineering [ICEE 2013], article 65998822013, 2013.

[4] T. Zhao, M. Wu, "Electric power characteristics of all-parallel at traction power supply system", Proceedings 2011 International Conference on Transportation, Mechanical, and Electrical Engineering [TMEE 2011], article 6199346, pp. 895-898, 2011.

[5] X.-H. Zhang, S.-Q. Wu, "Discussion and analysis of the metro environment control system using vehicle regenerative braking energy in cold region", Journal of Railway Engineering Society, vol. 33 , iss. 2, pp. 91-95, 2016.

[6] T. Li, M. Wu, F. He, K. Song, "Calculations of leakage impedance of rail to earth in ballastless track by finite element method", Lecture Notes in Electrical Engineering, vol. 288, iss. 2, pp. 223-232, 2014.

[7] V. V. Shevchenko, N. V. Arzamaszev, N. S. Bodruhina, "Elektroshabzhenie nazemnogo gorodskogo transporta" ["Power supply of city surface transit"], Transport, Moscow, 1987.
[8] V. I. Sopov, Y. A. Prokushev, "Electrosnabzhenie elektricheskogo transporta" ["Power supply of electric transport"], Novosibirsk State Technical University, Novosibirsk, 2006.

[9] W. Liu, Q. Li, B. Tang, M. Chen, "Probabilistic load flow for urban rail traction power supply based on Monte Carlo simulation", Journal of Southwest Jiaotong University, vol. 45, iss. 4, pp. 561-567, 2010.

[10] M. Wu, "Uniform chain circuit model for traction networks of electric railways", Proc. of the Chinese Society of Electrical Engineering, vol. 30 